\title{
Avaliação do preconceito contra diversidade sexual e de gênero: construção de um instrumento
}

\author{
Evaluation of prejudice against sexual and gender \\ diversity: Construction of an instrument
}

\author{
Angelo Brandelli COSTA ${ }^{1}$ \\ Denise Ruschel BANDEIRA ${ }^{1}$ \\ Henrique Caetano NARDI ${ }^{2}$
}

\section{Resumo}

O Brasil carece de ferramentas para avaliar o preconceito contra a diversidade sexual e de gênero, sendo necessários instrumentos com boas evidências de validade e confiabilidade e, principalmente, adaptados para o contexto brasileiro. Uma vez que o preconceito está fortemente associado à cultura e que no Brasil o preconceito contra orientações não heterossexuais está especialmente relacionado com expressões de gênero discordantes das hegemônicas, este estudo propõe a criação de um instrumento construído para as especificidades do contexto brasileiro, com evidências de validade e confiabilidade. Participaram do estudo 800 sujeitos, moradores de áreas rurais e urbanas do sul do Brasil, os quais responderam ao questionário Escala de Preconceito contra Diversidade Sexual e de Gênero. Foram conduzidas análises a partir da Teoria de Resposta ao Item, de validade de critério e de correlação com outro instrumento que avalia o mesmo construto. A escala apresentou boas evidências de validade e fidedignidade, bem como contextualização para a realidade brasileira, sugerindo que se trata de uma ferramenta útil para avaliar o preconceito.

Palavras-chave: Avaliação psicológica; Diversidade de gênero; Diversidade sexual; Preconceito.

\begin{abstract}
In Brazil there is a lack of tools to assess such prejudices with good evidences of validity and reliability, and, especially, contextualized to the Brazilian context. Since prejudice is strongly associated to the culture and that in Brazil prejudice against non-heterosexual orientations is especially related hegemonic discordant gender expressions we created an instrument. The overall objective of this article is to establish evidences of validity and reliability for this instrument developed for the specificities of the Brazilian reality. Eight-hundred respondents from rural and urban areas of southern Brazil completed the Scale of Prejudice Against Sexual and Gender Diversity. Analyzes were conducted using Item Response Theory, criterion validity, and relationship with an instrument that measures the same construct. The scale showed good validity and reliability, and adaptability for the Brazilian reality, suggesting that it is a useful tool to assess prejudice.
\end{abstract}

Keywords: Psychological assessment; Gender diversity; Sexual diversity; Prejudice.

$\boldsymbol{\nabla} \boldsymbol{\nabla} \boldsymbol{\nabla}$

1 Universidade Federal do Rio Grande do Sul, Departamento de Psicologia do Desenvolvimento e da Personalidade, Programa de Pós-Graduação em Psicologia. R. Ramiro Barcelos, 2600, Santa Cecília, 90035-003, Porto Alegre, RS, Brasil. Correspondência para/Correspondence to: A.B. COSTA. E-mail: <brandelli.costa@ufrgs.br>.

2 Universidade Federal do Rio Grande do Sul, Departamento de Psicologia Social e Institucional, Programa de Pós-Graduação em Psicologia Social e Institucional. Porto Alegre, RS, Brasil.

Apoio: Conselho Nacional de Desenvolvimento Científico e Tecnológico (Universal 9/2011 e Universal 14/2011). 
O termo homofobia foi introduzido por George Weinberg em 1972, ao publicar o livro "Society and the healthy homosexual" ("A sociedade e o homossexual saudável"). A expressão foi amplamente adotada por ativistas e incorporada pela Academia, especialmente dentro do campo de estudos do preconceito (Young-Bruehl, 1996). Embora o termo seja também amplamente utilizado pelos movimentos sociais, ele é hoje compreendido como um preconceito contra orientações não heterossexuais, ou seja, como uma atitude contra um grupo ou seus membros, que cria ou mantém uma relação hierárquica de status (Dovidio, Hewstone, Gilck, \& Esses, 2010).

Desde sua conceptualização, inúmeros instrumentos foram desenvolvidos para avaliar o preconceito contra orientações não heterossexuais, como "Homophobia Scale, Attitudes Toward Homosexuality Scale, Index of Homophobia", entre outros trinta instrumentos descritos por Schawanberg (1993). A maioria desses instrumentos apresenta problemas no seu desenvolvimento, uma vez que não apresentam evidências de validade e fidedignidade; utilizam amostras por conveniência, geralmente com estudantes universitários; alguns instrumentos apresentam problemas na definição do construto avaliado; e muitos estudos utilizam como medida de validade convergente instrumentos que avaliam aspectos que não estão teoricamente ligados à esse tipo de preconceito ( $\mathrm{O}^{\prime}$ Donohue \& Caselles, 1993; Schawanberg,1993). Costa, Bandeira e Nardi (2013a) atualizaram a revisão feita por Schawanberg e mostraram franco avanço na qualidade psicométrica dos instrumentos, além de constatarem que a maioria deles provinha do contexto norte-americano e/ou utilizava, quando oriundo de outros países, um conceito sem adaptação para as realidades locais e os contextos culturais específicos. do estudos empíricos sobre o preconceito contra orientações não heterossexuais no Brasil, percebeu-se que é necessário levar em consideração não apenas a orientação sexual, mas também as expressões de gênero, o que parece explicar a forma predo164 minante da apresentação do preconceito (Costa,
Peroni, Bandeira, \& Nardi, 2013b). Ou seja, apesar de existir conceitualmente uma distinção entre orientação sexual e expressão de gênero, do ponto de vista da manifestação do preconceito, essa distinção parece ser mais tênue. Nesse sentido, no Brasil, não se pode falar de preconceito contra orientações não heterossexuais sem levar em conta o preconceito contra expressões de gênero que não estão em conformidade às hegemônicas. Esse aspecto indica que, no contexto brasileiro, um homem homossexual ou heterossexual que apresenta atitudes e comportamentos ou se expressa de forma culturalmente considerada como feminina será alvo preferencial de preconceito, o mesmo ocorrendo com as mulheres quando se expressarem ou assumirem atitudes consideradas como masculinas.

A relação entre orientação sexual e expressões de gênero já foi discutida teoricamente por Warner (1991) quando desenvolveu o conceito de heteronormatividade, assim como por Rich (1994) ao cunhar o termo heterossexualidade compulsória. Entretanto, essa relação não foi incorporada pelos instrumentos disponíveis para avaliar o preconceito.

Em revisão sistemática de estudos empíricos, a presente pesquisa reforçou essa relação como uma característica clara no contexto brasileiro, indicando a necessidade da criação de um instrumento que incluísse tanto aspectos relativos à orientação sexual como às expressões de gênero. Muito embora tenham sido desenvolvidos diversos instrumentos para avaliar o preconceito contra orientações não heterossexuais, eles não levam em consideração essa especificidade da forma de apresentação do construto.

Marinho, Marques, Almeida, Menezes e Guerra (2004) adaptaram para o Brasil o instrumento de homofobia implícita e explícita proposto por Castillo, Rodríguez, Torres, Pérez e Martel (2003). A escala, desenvolvida originalmente em língua espanhola, consiste em uma subescala de homofobia explícita, com dez itens, e outra de homofobia implícita, com sete itens. Cada subescala possui dois fatores: a de homofobia explícita, rechaço e intimidade; e a de homofobia implícita, diferença de valores e sentimentos positivos. Participaram da versão brasileira do estudo 231 estudantes de uma 
universidade pública da Paraíba. Corroborou-se a existência das duas expressões de homofobia, explícita e implícita, apesar de não terem sido encontradas diferenças significativas entre os fatores de cada uma das subescalas.

Outro instrumento desenvolvido no contexto brasileiro busca a atribuição de adjetivos positivos e negativos a indivíduos hetero e homossexuais (Fleury \& Torres, 2007). A amostra foi composta por 135 estudantes de pós-graduação, graduados em Psicologia e em Administração de Empresas. Foram utilizados 24 adjetivos a partir de grupos de traços, sendo 12 traços naturais e 12 culturais, 12 positivos e 12 negativos. Os participantes foram solicitados a informar o grau em que a sociedade brasileira utilizaria os adjetivos da escala para caracterizar os homossexuais e os heterossexuais. A atribuição de características positivas aos heterossexuais foi significativamente superior à atribuição feita para os homossexuais.

A Escala de Atração Sexual e de Orientação Sexual e Escala de Crenças sobre Homossexualidade (ECH) (Cerqueira-Santos, Winter, Salles, Longo, \& Teodoro, 2007) foi testada em uma amostra de 442 universitários, oriundos de cursos das Ciências Humanas e Sociais (24,0\%), das Ciências Exatas (29,0\%) e das Ciências Biológicas e Saúde (47,0\%). O instrumento é composto de um questionário com 29 itens, com afirmações positivas e negativas sobre a homossexualidade masculina e feminina. Análises fatoriais mostraram um modelo de três fatores: representação negativa de gays e lésbicas $(\alpha=0,89)$; representação positiva $(\alpha=0,69)$; e diferenciação intergrupal entre gays e lésbicas $(\alpha=0,69)$. A solução fatorial explicou $45,7 \%$ da variância.

Por fim, Pulerwitz e Barker (2008) adaptaram para o Brasil a Gender-Equitable Men Scale (GEM Scale). A escala avalia as atitudes de jovens adultos homens em relação às normas de gênero. A pesquisa foi realizada com 742 homens de diferentes contextos sociais: uma favela, um bairro de classes baixa e média-baixa, e um bairro de classes média e alta. Participaram da pesquisa 223 homens com idade entre 15 e 24 anos. Concluiu-se que o instrumento apresentava boas evidências de validade e consistência interna.
Percebe-se que os instrumentos disponíveis no contexto brasileiro ou privilegiam a orientação sexual, ou as normas de gênero, não atendendo à forma como o preconceito se manifesta no Brasil conforme demonstrado por Costa et al. (2013b). Assim, o objetivo deste estudo foi construir e buscar evidências de validade e fidedignidade de um instrumento para avaliar esse tipo de preconceito, levando em conta as especificidades brasileiras.

\section{Método}

\section{Participantes}

Participaram da pesquisa 615 estudantes de Ensino Médio, 149 professores e 32 funcionários de seis escolas públicas gaúchas, nas cidades de Porto Alegre, Venâncio Aires e Santa Cruz do Sul. A amostra final consistiu em 800 participantes, sendo 301 homens e 499 mulheres. A idade média dos funcionários foi de 43,63 (Desvio-Padrão $D P=11,10)$, dos professores $41,45(D P=10,00) \mathrm{e}$ dos estudantes $17,39(D P=5,49)$. A amostra foi não probabilística, ou seja, tanto as escolas quanto os participantes foram recrutados por conveniência.

\section{Instrumentos}

\section{Medidas sociodemográficas}

Os participantes responderam a questões sociodemográficas sobre gênero, idade, status socioeconômico de acordo com o Critério de Classificação Econômica Brasil (Associação Brasileira de Empresas de Pesquisa, 2013), orientação sexual (heterossexual ou não), local de moradia (capital ou interior) e religiosidade (por meio da resposta à pergunta "possui religião?"). Além disso, investigando a hipótese do contato, os participantes foram questionados se tinham "amigos, parentes ou conhecidos próximos gays, lésbicas, travestis ou transexuais" (Herek \& Capitanio, 1996). Por fim, o acesso à informação foi medido por meio de cinco 
perguntas relativas à disponibilidade de rádio, jornais, revistas, Internet e televisão (Brasil, 2009).

\section{Distância social}

Como medida de critério para o preconceito foi utilizada uma escala de distância social de Bogardus, adaptada para investigar a relação com gays, lésbicas, travestis e transexuais (Rodrigues, 2009). Os participantes selecionaram as seguintes alternativas em relação a cada um dos grupos: 1) aceitaria como membro da minha família; 2) aceitaria como amiga; 3) aceitaria como colega de trabalho/escola; 4) aceitaria como vizinha; 5) aceitaria em meu bairro; 6) aceitaria em minha cidade; e 7) não aceitaria.

\section{Escala de Preconceito contra Diversidade Sexual e de Gênero}

A Escala de Preconceito contra Diversidade Sexual e de Gênero (EPDSG) foi composta por dois instrumentos: um avaliando preconceito contra Orientação Sexual (OS) e outro investigando preconceito contra não conformidade de Gênero e Transexualidade (GT).

A escala que avalia preconceito contra OS, Attitudes Toward Lesbians and Gays Scale (ATLG) (Herek, 1988), foi selecionada a partir de uma revisão sistemática que buscou evidências de validade e fidedignidade em instrumentos que avaliam homofobia e construtos correlatos (Costa et al., 2013b). A escala foi desenvolvida nos Estados Unidos por Gregory Herek, em 1988. Após contato com o autor e autorização para o uso da escala, decidiu-se utilizar a versão mais atual do instrumento (Herek \& McLemore, 2011). Trata-se de uma medida de dez itens, composta por duas subescalas, uma que avalia o preconceito contra lésbicas e outra contra gays. A correlação entre as duas subescalas é alta, 0,84, e o alfa de Cronbach total da escala é de 0,94 .

A escala Genderism and Transphobia Scale (Hill \& Willoughby, 2005) foi escolhida para avaliar 166 o preconceito contra não conformidade de gênero e transexualidade, dentre outras três identificadas em uma revisão sistemática não publicada. Essa escala foi selecionada por medir o preconceito tanto contra não conformidade de gênero quanto por identidade de gênero e transexualidade. O instrumento, desenvolvido nos Estados Unidos, é composto por 32 itens que formam dois fatores: genderismo/transfobia e violência de gênero. A correlação entre os fatores é de 0,85. O alfa de Cronbach do instrumento foi de 0,96 . Um painel de três pesquisadores expertos na área selecionou 11 itens da subescala genderismo/transfobia, os quais refletiam a natureza do fenômeno da forma como se apresenta no contexto brasileiro. Além disso, o painel incluiu a identidade de gênero travesti, e criou um novo item adaptado a partir de outro existente, com aprovação e autorização dos autores da escala.

Inicialmente, foi realizado o processo de tradução e adaptação das escalas do inglês para o português, por três juízes brasileiros fluentes na língua inglesa e experientes no campo de gênero e sexualidade. A partir do consenso entre juízes, foi produzida uma versão definitiva da tradução. A seguir, as escalas traduzidas foram enviadas para dois nativos norte-americanos, com experiência no campo de sexualidade e gênero para a retrotradução. Uma nova versão unificada foi enviada para os autores das escalas, que aceitaram a tradução e as adaptações produzidas, em processo que seguiu o modelo de adaptação sugerido por Borsa, Damásio e Bandeira (2012).

A escala final foi formada por 22 itens com afirmações múltiplas sobre o preconceito contra gays, lésbicas, não conformidade de gênero e transexualidade. Eles foram avaliados em uma escala de cinco pontos, sendo 1 para "discordo totalmente", e 5 para "concordo totalmente". Escores mais próximos a 5 evidenciavam maior preconceito.

\section{Procedimentos}

O presente artigo é fruto de um projeto aprovado pela Comissão de Pesquisa do Instituto de Psicologia da Universidade Federal do Rio Grande do Sul (Projeto $n^{\circ}$ 23459) e pelo Comitê de Ética em Pesquisa do mesmo Instituto (CAAE 
04642712.9.0000.5334). Em um primeiro momento, os diretores das escolas contatadas assinaram o Termo de Consentimento Institucional, autorizando a realização da pesquisa. Em seguida, o instrumento foi aplicado por pesquisadores treinados - individualmente no caso de funcionários e professores, e em grupo no caso dos estudantes. Depois de receberem informações sobre o propósito do estudo, os participantes foram convidados, a responder voluntariamente ao questionário autoaplicável. Os termos de consentimento foram assinados pelos participantes maiores de 18 anos ou, se menores, pelo seu responsável legal.

Não foram considerados os participantes que responderam a menos de $80,0 \%$ de cada um dos instrumentos que compuseram o protocolo de pesquisa. Com a exclusão desses casos, os dados omissos não chegaram a 1,8\% do total. Esses foram preenchidos através de imputação de dados por regressão. Foi observado que todos os itens do instrumento possuem padrão assimétrico. Os valores de assimetria (skewness) variaram de 0,08 até -1,09. Já os valores de achatamento (kurtosis) variaram de 0,17 até 1,48 . Além disso, não foi observada multicolinearidade entre as variáveis avaliadas.

Para avaliar as propriedades psicométricas da escala, foi conduzida uma análise de teoria de resposta ao item Rating Scale do modelo Rasch (Andrich, 1978), por meio do software Winsteps versão 3.72.2 (Linacre, 2011). O modelo permite estimar, em um mesmo contínuo linear simples, os parâmetros da dificuldade dos itens ( $\delta$ ) e o nível de traço latente (theta, $\theta$ ) das pessoas. Esses parâmetros são expressos em unidades de logaritmos de chance (log-odds units ou logits). A dimensionalidade dos itens foi investigada por meio da análise dos contrastes principais (análise de componentes principais dos resíduos do modelo), cujos autovalores <2 são indicadores de unidimensionalidade. A dependência local dos itens foi inspecionada por meio de suas correlações residuais, sendo recomendados valores $<0,3$. O ajuste dos itens foi avaliado por meio dos índices infit e outfit, que devem se situar entre 0,5 e 1,5. Por fim, foi investigado o Differential Item Functioning (DIF, Funcionamento Diferencial dos Itens, i.e., diferenças na probabilidade de endosso) em relação ao gênero, observando-se os valores do contraste da dificuldade dos itens entre os grupos $(<|0,5|$ logit) (Linacre, 2011).

O escore total da escala resultante das análises foi computado calculando-se a média dos escores dos itens. Foram realizados testes $t$ para estabelecer a diferença no escore total entre grupos de gênero (masculino ou feminino), religião (possui ou não possui), local de moradia (capital ou interior), nível de acesso à informação (alto ou baixo), possuir amigo LGBT (Lésbicas, Gays, Bissexuais, Travestis e Transexuais) (sim ou não) e orientação sexual (heterossexual ou não heterossexual). Além disso, correlações de Pearson foram realizadas entre as medidas de distância social e o valor total da escala. Consideraram-se significativas as diferenças obtidas $\operatorname{com} p<0,05$. O escore total de acesso à informação foi calculado por meio de média ponderada da resposta aos itens. Aqueles que apresentaram escore acima da média foram considerados como tendo alto acesso à informação, e aqueles com escore abaixo da média como tendo baixo acesso à informação. Para a realização dessas análises foi utilizado o pacote estatístico Statistical Package for the Social Sciences (SPSS) (versão 21).

\section{Resultados}

A análise dos contrastes principais evidenciou a presença de um componente de autovalor $=$ 2,6, explicando $12 \%$ da variância residual dos itens. Os itens que apresentaram cargas componenciais salientes foram: $8(0,73), 3(0,66), 10(0,55)$ e 5 $(0,45)$. A inspeção das correlações residuais indicou a presença de dependência local entre os seguintes pares de itens: $17 / 19(r=0,75), 3 / 8(r=0,73), 5 / 10$ $(r=0,66)$ e $4 / 9(r=0,64)$. A partir desses critérios, o conjunto de itens foi excluído das análises subsequentes, com exceção dos itens 4 e 19, que foram mantidos pelo critério de maior correlação item-theta. Os 16 itens restantes do instrumento mostraram-se unidimensionais e não apresentaram dependência local (Anexo). Seus parâmetros psicométricos são apresentados na Tabela 1. Considerando-se especialmente o critério de infit, que 
Tabela 1

Fidedignidade da Escala, Dificuldade e Ajuste dos Itens

\begin{tabular}{|c|c|c|c|c|c|c|}
\hline Itens & Fidedignidade dos itens & Dificuldade $\delta(E P)$ & Infit & Outfit & $\mathrm{DIF}^{*}$ & Fidedignidade das pessoas \\
\hline & 0,98 & & & & & 0,77 \\
\hline & $\alpha=0,93$ & & & & & \\
\hline 5 & & $0,31(0,04)$ & 1,14 & 1,13 & 0,50 & \\
\hline 8 & & $0,29(0,04)$ & 1,54 & 1,88 & 0,13 & \\
\hline 11 & & $0,29(0,04)$ & 0,73 & 0,66 & 0,04 & \\
\hline 3 & & $0,20(0,04)$ & 0,86 & 0,92 & $-0,08$ & \\
\hline 13 & & $0,18(0,04)$ & 0,91 & 0,91 & 0,00 & \\
\hline 2 & & $0,14(0,04)$ & 0,85 & 0,82 & $-0,30$ & \\
\hline 10 & & $0,08(0,04)$ & 1,50 & 1,48 & $-0,34$ & \\
\hline 14 & & $0,05(0,04)$ & 0,92 & 0,80 & $-0,53$ & \\
\hline 4 & & $0,04(0,04)$ & 1,03 & 1,08 & 0,65 & \\
\hline 16 & & $-0,01(0,03)$ & 1,07 & 1,15 & 0,17 & \\
\hline 7 & & $-0,04(0,03)$ & 0,69 & 0,63 & $-0,18$ & \\
\hline 12 & & $-0,09(0,03)$ & 0,95 & 0,99 & 0,00 & \\
\hline 1 & & $-0,20(0,03)$ & 0,87 & 1,01 & 0,08 & \\
\hline 9 & & $-0,20(0,03)$ & 0,83 & 0,99 & 0,15 & \\
\hline 6 & & $-0,31(0,03)$ & 0,82 & 0,85 & $-0,16$ & \\
\hline 15 & & $-0,74(0,03)$ & 1,61 & 1,72 & $-0,10$ & \\
\hline Média & & 0,00 & 1,02 & 1,06 & 0,00 & \\
\hline \multirow[t]{2}{*}{ (Desvio-Padrão) } & & $(0,26)$ & $(0,28)$ & $(0,34)$ & $(0,29)$ & \\
\hline & & & & & $\Sigma=0,03$ & \\
\hline
\end{tabular}

Nota: *Funcionamento diferencial, categoria de referência: homem.

EP: Erro-Padrão; $\delta$ : mi/dificuldade; $\alpha$ : alpha de Cronbach; $\sum$ : somatório; DIF: Differential Item Functioning.
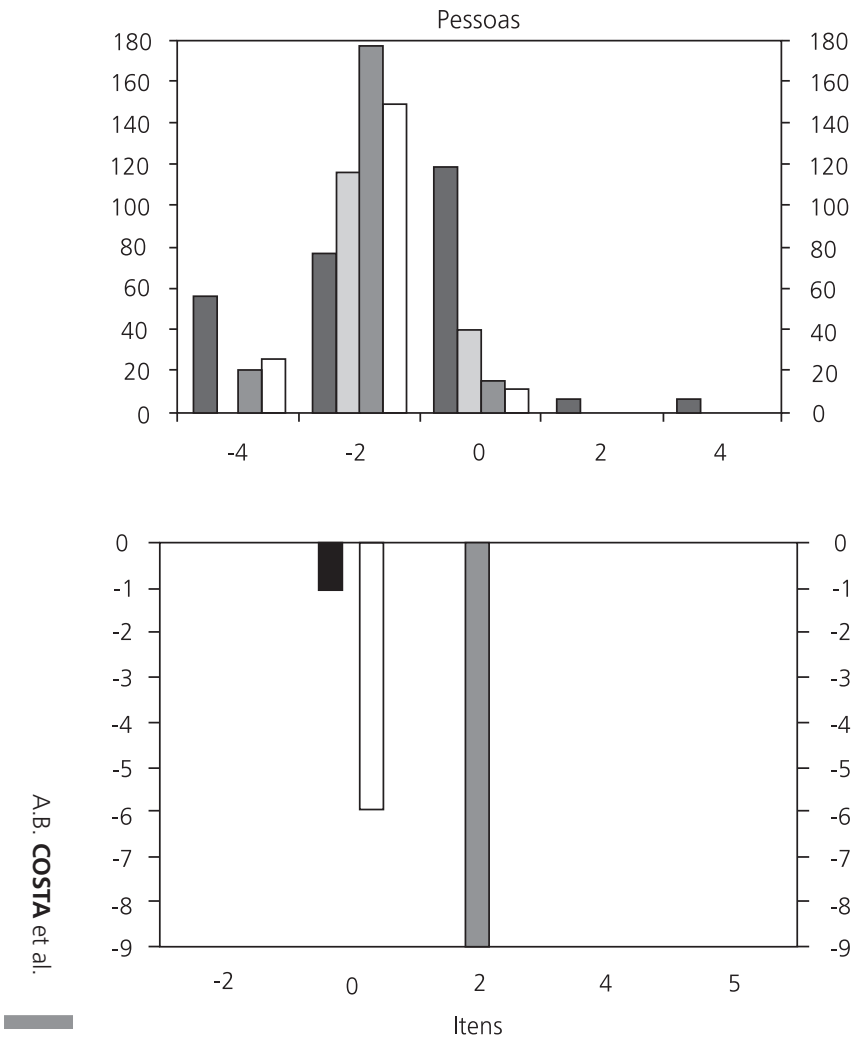

168

Figura 1. Mapa das pessoas e itens. reflete a congruência entre nível de habilidade e dificuldade do item, os itens foram considerados ajustados ao modelo de mensuração. Apesar de os itens 6, 7 e 20 apresentam valores de DIF acima do recomendado $(<|0,5|$ logit $)$, tendo em vista respondentes homens e mulheres, os valores da média e o somatório dos contrastes próximos a zero indicam que a escala apresenta certo equilíbrio em relação ao número de itens que favorecem cada um dos grupos, i.e., são mais facilmente endossados (Linacre, 2011). A Figura 1 representa a distribuição das pessoas e itens no contínuo logit. É possível observar que os itens estão agrupados no centro da escala logit, enquanto a maior parte dos participantes encontra-se na porção negativa do contínuo, indicando baixos níveis no traço em questão (Média $-M \theta=-0,83, D P=1,19$ ).

O valor total da escala EPDSG foi calculado com média ponderada dos escores dos itens. As diferenças entre as médias dos grupos sociodemográficos foram calculadas utilizando testes $t$ (Tabela 2). A análise indica que os homens são mais preconceituosos do que as mulheres, $t(571)=10,52$, 
Tabela 2

Diferença de médias do preconceito total entre grupos sociodemográficos

\begin{tabular}{|c|c|c|c|c|c|}
\hline Variável & $n$ & $M$ & $D P$ & IC95\% & $p$ \\
\hline \multicolumn{6}{|l|}{ Gênero } \\
\hline Masculino & 301 & 2,64 & 1,00 & $2,53,2,75$ & $<0,001$ \\
\hline Feminino & 499 & 2,01 & 0,87 & $1,93,2,09$ & \\
\hline \multicolumn{6}{|l|}{ Religião } \\
\hline Possui & 595 & 2,34 & 0,98 & $2,26,2,42$ & $<0,001$ \\
\hline Não possui & 205 & 1,99 & 0,88 & $1,86,2,11$ & \\
\hline \multicolumn{6}{|l|}{ Local de moradia } \\
\hline Capital & 478 & 2,08 & 0,95 & $2,00,2,17$ & $<0,001$ \\
\hline Interior & 322 & 2,50 & 0,94 & $2,39,2,60$ & \\
\hline \multicolumn{6}{|c|}{ Nível de acesso à informação } \\
\hline Alto & 736 & 2,22 & 0,97 & $2,15,2,30$ & $<0,02$ \\
\hline Baixo & 64 & 2,52 & 0,92 & $2,29,2,75$ & \\
\hline \multicolumn{6}{|l|}{ Amigo $L G B T$} \\
\hline Sim & 633 & 2,08 & 0,90 & $2,01,2,15$ & $<0,001$ \\
\hline Não & 167 & 2,87 & 0,94 & $2,73,3,01$ & \\
\hline \multicolumn{6}{|l|}{ Orientação sexual } \\
\hline Heterossexual & 738 & 2,26 & 0,95 & $2,19,2,33$ & $<0,001$ \\
\hline Não heterossexual & 35 & 1,51 & 0,69 & $1,28,1,75$ & \\
\hline Total & 800 & 2,25 & 0,97 & $2,18,2,31$ & \\
\hline
\end{tabular}

Nota: M: Média; DP: Desvio-Padrão; IC95\%: Intervalo de Confiança de 95\%; LGBT: Lésbicas, Gays, Bissexuais, Travestis e Transexuais.

$p<0,001$ - assim como os que possuem religião, $t(390)=7,36, p<0.001$; os que moram no interior, $t(798)=0,02, p<0,001$; os participantes com baixo acesso à informação, $t(798)=0,26, p<0,02$; e os que não têm amigos ou conhecidos LGBT, $t(798)=0,23, p<0,001$. Além disso, existe diferença de grau de preconceito por orientação sexual do participante $t(40,37)=11,78, p<0,001$. Os resultados corroboram a direção das hipóteses consagradas na literatura, apontando a capacidade discriminante da escala. Além disso, a escala EPDSG apresentou correlações positivas com as escalas de distância social, seja com gays $p<0,01(r=0,64)$, lésbicas $p<0,01(r=0,53)$ ou travestis e transexuais $p<0,01(r=0,65)$, demostrando a capacidade convergente da medida conforme a Tabela 3.

\section{Discussão}

A criação e a validação de um instrumento para avaliar o preconceito contra a diversidade
Tabela 3

Correlação entre o escore total da escala e a distância social

\begin{tabular}{lcccc}
\hline Variável & 1 & 2 & 3 & 4 \\
\hline 1. Preconceito total & - & $0,64^{*}$ & $0,53^{*}$ & $0,65^{*}$ \\
2. Gays & & - & $0,72^{*}$ & $0,73^{*}$ \\
3. Lésbicas & & - & $0,61^{*}$ \\
4. Travestis e transexuais & & & - \\
\hline Nota: ${ }^{*} p<0,01$. & & &
\end{tabular}

sexual e de gênero foi o objetivo deste estudo. Durante esse processo, utilizaram-se itens oriundos de dois instrumentos desenvolvidos para avaliar preconceito contra diversidade sexual e de gênero, além inclusão da identidade de gênero travesti e da criação de um item a fim de deixar o instrumento mais próximo da realidade brasileira. Buscou-se também seguir um padrão rigoroso no processo de adaptação ao contexto local. Realizaram-se análises a partir da Teoria de Resposta ao Item, de diferenciação de grupos critério e de correlação com outro instrumento que mensura o mesmo construto. 
As análises mostraram que os itens que compõem a escala de preconceito contra OS e aqueles que formam a escala de preconceito contra não conformidade de GT avaliam uma única dimensão, aqui denominada Preconceito Contra a Diversidade Sexual e de Gênero. Alguns itens originalmente propostos apresentaram cargas componenciais salientes, o que sugeriu sua exclusão: "A homossexualidade masculina é uma expressão natural da sexualidade em homens"; "A homossexualidade feminina é uma expressão natural da sexualidade nas mulheres"; "A homossexualidade feminina é apenas um tipo diferente de estilo de vida que não deve ser condenado"; e "A homossexualidade masculina é apenas um tipo diferente de estilo de vida que não deve ser condenado".

A hipótese desta pesquisa, explicando a não congruência desses itens com o restante da escala, é que o amplo debate sobre as origens da homossexualidade (natureza versus cultura) formaria grupos independentes com base em suas crenças. Isso significa que a crença de que a homossexualidade é natural (origem biológica) ou que é um estilo de vida (origem social) independe do grau de preconceito do respondente. Esse padrão de resposta até pode estar relacionado com as definições de "natural" e "estilo de vida" na língua portuguesa, mas os autores da presente pesquisa tendem a pensar que, hoje, no Brasil, as crenças sobre a origem da homossexualidade independem do preconceito, o que justifica a exclusão daqueles itens.

A partir dos processos de adaptação e validação realizados, a escala em sua versão final foi composta por 16 itens (Anexo) que, conjuntamente, indicam a pontuação geral do instrumento, salientando-se que o item 15 deve ter a pontuação invertida. Todavia, deve-se considerar a possibilidade de uma avaliação item a item, visto que os critérios elencados por cada um deles podem produzir informações importantes relativas à especificidade do preconceito contra lésbicas, gays, transexuais e não conformidade de gênero. A relação entre preconceito contra diversdade sexual e de gênero já vem sendo apontada em estudos empíricos conduzidos no Brasil (Costa et al., 2013b). Apesar de tal constatação ser reforçada pelo pre170 sente estudo, ela merece maiores investigações, a fim de verificar a natureza do construto e suas relações.

Ao longo dos anos, alguns consensos foram construídos a respeito do preconceito contra diversidade sexual e de gênero: a) pessoas que vivem em grandes centros urbanos tendem a ser menos preconceituosas que aquelas que vivem no interior; b) mulheres tendem a ser menos preconceituosas do que homens; c) o maior acesso à informação está associado a menor grau de preconceito; d) o preconceito correlaciona-se com a religiosidade; e) pessoas que tiveram contato prévio com indivíduos não heterossexuais tendem a ser menos preconceituosas do que aquelas que nunca tiveram; f) indivíduos não heterossexuais são menos preconceituosos do que os heterossexuais (Dovidio et al., 2010; Herek, 2000). Constatou-se que o instrumento conseguiu diferenciar grupos critérios a partir desses consensos. Além disso, o instrumento apresentou convergência com a escala de distância social, medida clássica para avaliação do preconceito.

Assim, pôde-se concluir que a escala de preconceito contra a diversidade sexual e de gênero apresenta boas evidências de validade e fidedignidade, além de responder ao objetivo de estar adaptada à forma como o preconceito se apresenta no Brasil, sugerindo que se trata de uma ferramenta útil para o contexto local. Sua utilização pode promover ganhos metodológicos importantes nos estudos sobre preconceito realizados no Brasil, uma vez que muitos se baseiam em medidas não sistemáticas ou não adaptadas para a realidade local.

Apesar do cuidado com o processo de adaptação e validação do instrumento, algumas limitações devem ser apontadas. Os itens estão agrupados no centro da escala logit, de modo que se sugere a realização de novos estudos a fim de se buscarem itens mais sensíveis, que possam avaliar tanto o preconceito mais sutil quanto o mais flagrante, a partir de uma versão revisada do presente instrumento.

\section{Referências}

Associação Brasileira de Empresas de Pesquisa. (2013). Critério de Classificação Econômica Brasil. Recuperado 
em outubro 10, 2013, disponível em http://www. abep.org/novo/FileGenerate. ashx?id=297

Andrich, D. (1978). A rating formulation for ordered response categories. Psychometrika, 43(4), 561-573. http://dx.doi.org.br/10.1007/BF02293814

Borsa, J. C., Damásio, B. F., \& Bandeira, D. R. (2012). Adaptação e validação de instrumentos psicológicos entre culturas: algumas considerações. Paidéia, 22(53), 423-432. http://dx.doi.org.br/10.1590/S0103-86 3X2012000300014

Brasil. Ministério da Educação, Instituto Nacional de Estudos e Pesquisas Educacionais, \& Fundação Instituto de Pesquisas Econômicas. (2009). Pesquisa sobre discriminação e preconceito no ambiente escolar. Brasília: Ministério da Educação. Recuperado em outubro 10, 2013, de http://www.dominiopublico.gov.br/ download/texto/me04651a.pdf

Castillo, M., Rodríguez, V., Torres, R., Pérez, A., \& Martel, É. (2003). La medida de la homofobia manifesta y sutil. Psicothema, 15(2), 197-204.

Cerqueira-Santos, E., Winter, F. D. S., Salles, L. A., Longo, J. L., \& Teodoro, M. L. (2007). Contato interpessoal e crenças sobre homossexualidade: desenvolvimento de uma escala. Interação em Psicologia, 11(2), 221-229.

Costa, A. B., Bandeira, D. R., \& Nardi, H. C. (2013a). Systematic review of instruments measuring homophobia and related constructs. Journal of Applied Social Psychology, 43(6), 1324-1332. http://dx.doi.org.br/ 10.1111/jasp. 12140

Costa, A. B., Peroni, R. O., Bandeira, D. R., \& Nardi, H. C. (2013b). Homophobia or sexism? A systematic review of prejudice against nonheterosexual orientation in Brazil. International Journal of Psychology, 48(5), 900909. http://dx.doi.org.br/10.1080/00207594.2012.72 9839

Dovidio, J., Hewstone, M., Gilck, P., \& Esses, V. (2010). The SAGE handbook of prejudice, stereotyping and discrimination. London: SAGE.

Fleury, A. R. D., \& Torres, A. R. R. (2007). Análise psicossocial do preconceito contra homossexuais. Estudos de Psicologia (Campinas), 24(4), 475-486. http:// dx.doi.org.br/10.1590/S0103-166X2007000400007

Herek, G. M. (1988). Heterosexuals' attitudes toward lesbians and gay men: Correlates and gender differences. Journal of Sex Research, 25(4), 451-477. http://dx.doi.org.br/10.1080/00224498809551476

Herek, G. M. (2000). The psychology of sexual prejudice. Current Directions in Psychological Science, 9(1), 19-22. http://dx.doi.org.br/10.1111/1467-8721.00051
Herek, G. M., \& Capitanio, J. P. (1996). Some of my best friends: Intergroup contact, concealable stigma, and heterosexuals attitudes toward gay men and lesbians. Personality and Social Psychology Bulletin, 22(4), 412-424. http://dx.doi.org.br/10.1177/0146167296 224007

Herek, G. M., \& McLemore, K. A. (2011). The Attitudes Toward Lesbians and Gay Men (ATLG) scale. In T. Fisher, C. M. Davis, W. L. Yarber, \& S. L. Davis (Eds.), Handbook of sexuality-related measures (pp.415-417). Oxford: Taylor \& Francis.

Hill, D. B., \& Willoughby, B. L. (2005). The development and validation of the genderism and transphobia scale. Sex Roles, 53(7/8), 531-544. http://dx.doi.org.br/ 10.1007/s11199-005-7140-x

Linacre, J. M. (2011). A user's guide to WINSTEPS/ MINISTEP Rasch-model computer programs. Chicago: MESA Press.

Marinho, C. D. A., Marques, E. F., Almeida, D. R. D., Menezes, A. R. D., \& Guerra, V. M. (2004). Adaptação da escala de homofobia implícita e explícita ao contexto brasileiro. Paidéia, 14(29), 371-379.

Pulerwitz, J., \& Barker, G. (2008). Measuring attitudes toward gender norms among young men in Brazil development and psychometric evaluation of the GEM scale. Men and Masculinities, 10(3), 322-338. http:// dx.doi.org.br/10.1177/1097184X06298778

O'Donohue, W., \& Caselles, C. E. (1993). Homophobia: Conceptual, definitional, and value issues. Journal of Psychopathology and Behavioral Assessment, 15(3), 177-19. http://dx.doi.org.br/10.1007/BF01371377

Rich, A. (1994). Compulsory heterosexuality and lesbian existence. In A. Rich Blood, bread, and poetry (pp.65-87). New York: Norton Paperback.

Rodrigues, A. (2009). Psicologia social. Petrópolis: Vozes.

Schawanberg, S. L. (1993). Attitudes towards gay man and lesbian woman: Instrumentation issues. Journal of Homosexuality, 26(1), 99-136.

Warner, M. (1991) Fear of a queer planet. Durham: Duke University Press.

Weinberg, G. (1972). Society and the healthy homosexual. New York: St. Martin's Press.

Young-Bruehl, E. (1996). The anatomy of prejudices. Cambridge: Harvard University Press.

Recebido: setembro 27, 2013

Versão final: dezembro 19, 2013

Aprovado: março 13, 2014 
ANEXO

ESCALA DE PRECONCEITO CONTRA DIVERSIDADE SEXUAL E DE GÊNERO

Marque como você responderia às seguintes afirmativas utilizando a escala de cinco opções descrita abaixo. Por favor, responda CUIDADOSAMENTE e HONESTAMENTE a cada pergunta. É importante indicar como você se sente AGORA e não como você pode ter-se sentido no PASSADO. Algumas das situações podem ser estranhas para você, mas tente pensar sobre situações semelhantes que você possa ter vivenciado. Responda a cada item e não se preocupe com suas respostas anteriores. Não há respostas certas ou erradas.

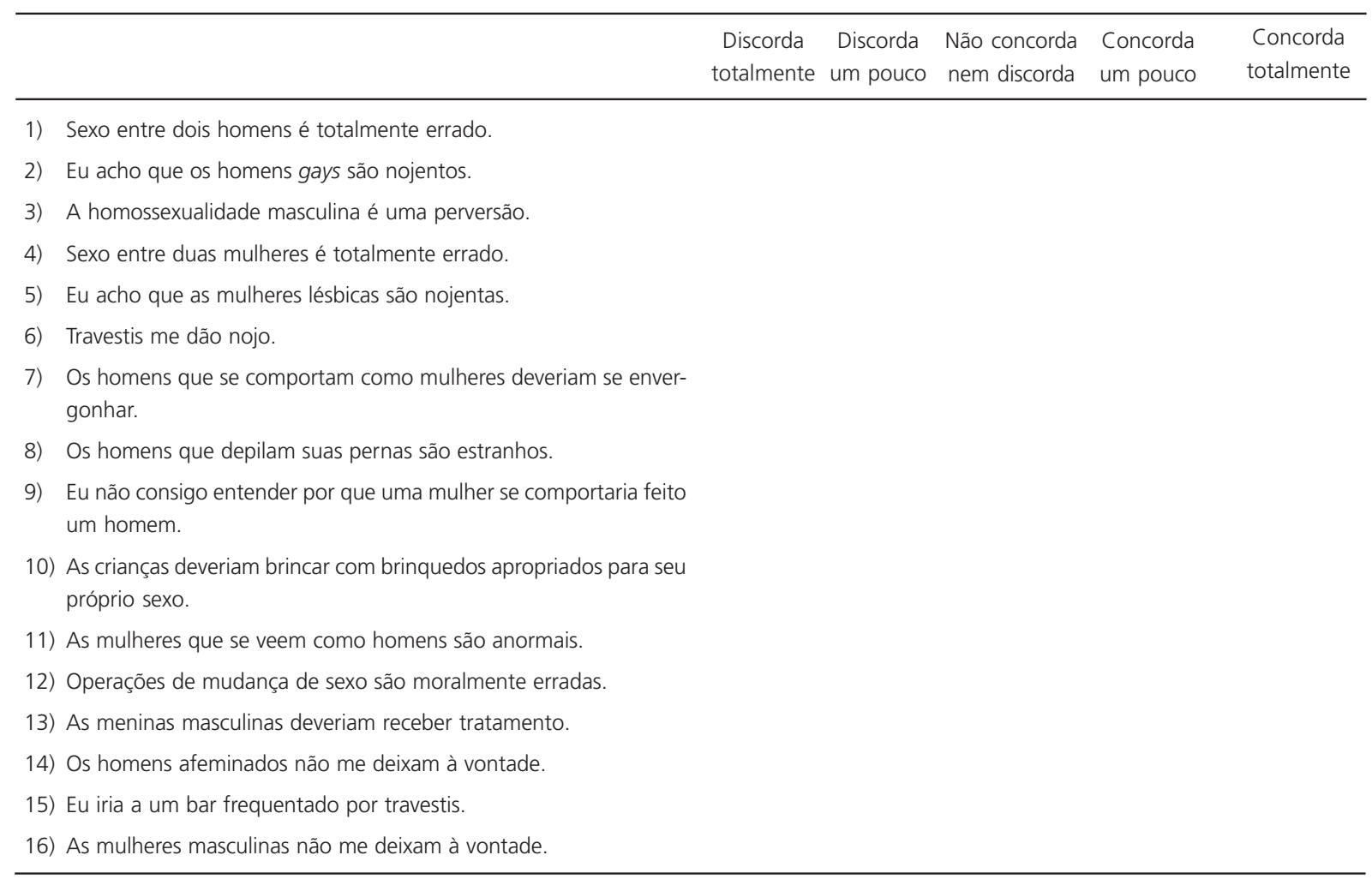

172 